\title{
Validação do Modelo Dinâmico de um Atuador Eletromagnético Linear Tubular Utilizando Cossimulação com Método dos Elementos Finitos
}

\author{
Ben Hur Bandeira Boff*, Jeferson Vieira Flores* e \\ Paulo Roberto Eckert* \\ *Programa de Pós-graduação em Engenharia Elétrica da UFRGS (PPGEE-UFRGS), \\ Porto Alegre, RS 90035-190,Brasil (e-mail: bandeira.boff@ufrgs.br, jeferson.flores@ufrgs.br, paulo.eckert@ufrgs.br)
}

\begin{abstract}
This work addresses the dynamic model validation of a three-phase synchronous tubular linear electromagnetic actuator with field excitation by permanent magnets. Two state-space models, a nonlinear and a linearized around an operation point, are developed considering the synchronous reference frame. For validation purposes, a cosimulation scheme is proposed in which the drive circuit of the actuator is performed in the ANSYS Electromagnetics ${ }^{\circledR}$ Twin Builder software and the finite element model of the electromagnetic device is described in ANSYS Electromagnetics ${ }^{\circledR}$ Maxwell. The results are analyzed in two situations: quadrature voltage step and disturbance load force step. This approach allows evaluating the proposed analytical models against non-idealities of the electromagnetic device such as saturation, leakage flux, armature reaction and end effects without the need for experimental validation.
\end{abstract}

\begin{abstract}
Resumo: Este trabalho tem como objetivo a validação do modelo dinâmico de um atuador eletromagnético linear tubular trifásico síncrono com excitação de campo por ímãs permanentes. Dois modelos em espaço de estados, um não linear e outro linearizado no entorno de um ponto de equilíbrio, foram desenvolvidos considerando um referencial de coordenadas síncronas. Para fins de validação, é proposto um ambiente de cossimulação onde o acionamento do atuador é realizado no ANSYS Electromagnetics ${ }^{\circledR}$ Twin Builder e o modelo em elementos finitos do dispositivo eletromagnético é descrito no ANSYS Electromagnetics ${ }^{\circledR}$ Maxwell. Os resultados são analisados a partir de dois cenários: degrau de tensão em quadratura e degrau de distúrbio na força de carga. Esta abordagem permite que os modelos analíticos sejam avaliados frente a não idealidades do dispositivo eletromagnético tais como saturação, fluxos dispersos, reação de armadura e efeitos de extremidade sem a necessidade de validação experimental.
\end{abstract}

Keywords: Cosimulation; Finite element model; Linear electromagnetic actuator; Model validation; Industry applications; Permanent magnet synchronous machine.

Palavras-chaves: Aplicações na indústria; Atuador eletromagnético linear; Cossimulação; Máquina síncrona de ímãs permanentes; Método dos elementos finitos; Validação do modelo.

\section{INTRODUÇÃO}

Atuadores eletromagnéticos lineares são máquinas elétricas com movimentação axial, construídas para diferentes aplicações em áreas como medicina, indústria aeroespacial, automotiva, entre outras. Em vista da enorme gama de aplicações para esses atuadores, existe um grande número de topologias criadas para se adequar à necessidade específica do projeto e/ou também viabilizar a construção de um protótipo. As topologias mais comuns de atuadores são a de indução, de relutância, síncrono de ímãs permanentes com ou sem ranhuras e síncrono com arranjo de quase-Halbach (Boldea, 2013; Eckert et al., 2018). Estudos recentes têm se focado nesta última categoria, mais especificamente em atuadores síncronos de ímãs permanentes com duplo arranjo de quase-Halbach e bobina longa trifásica (Eckert et al., 2016; Zanatta et al., 2018). Esta topologia possui algumas vantagens quando comparada às outras, como alta densidade de força, pequenas perdas magnéticas, pequena massa móvel e força de relutância praticamente nula (Eckert et al., 2018).

A aplicação de controle em máquinas elétricas exige além de informações de parâmetros elétricos e mecânicos, o conhecimento do seu modelo dinâmico. A técnica mais usual de modelagem de máquinas lineares é baseada na equivalência entre as dinâmicas das máquinas rotativas e lineares (Boldea, 2013).

Considerando as etapas iniciais de projeto, é comum que o comportamento do sistema seja verificado em simulação antes da sua aplicação efetiva na planta real. No contexto de máquinas síncronas lineares, esta etapa é comumente realizada com o auxílio de métodos numéricos em elementos finitos. Para o caso particular do atuador avaliado neste trabalho, a etapa de projeto é apresentada em (Eckert et al., 2016). Modelos analíticos e semianalíticos também são ferramentas utilizadas para a descrição de grandezas eletromagnéticas dos dispositivos, como, por exemplo, em 
(Eckert et al., 2018) e (Boff et al., 2017), onde esses modelos são validados experimentalmente.

Do ponto de vista da modelagem dinâmica de dispositivos eletromagnéticos, um recurso bastante útil é a cossimulação transiente entre um simulador de circuitos e o modelo da máquina em elementos finitos. O simulador de circuitos é responsável por proporcionar o circuito de acionamento adequado para o modelo em elementos finitos, sendo possível a obtenção de resultados eletromagnéticos dinâmicos. Essa metodologia é aplicada em (Zhu et al., 2007; Liang et al., 2014) para avaliação de características específicas em máquinas elétricas rotativas, e em (Fang et al., 2015) onde um dispositivo eletromagnético de ímãs permanentes é avaliado em relação ao seu tempo de resposta para aplicações em disjuntores a vácuo. Apesar desses estudos validando parâmetros dinâmicos, não foram encontrados trabalhos envolvendo uma cossimulação transiente com atuadores lineares para validação de parâmetros dinâmicos do modelo relacionados à movimentação para posterior projeto de controle.

Este trabalho propõe a validação do modelo dinâmico no espaço de estados de um atuador linear utilizando um ambiente de cossimulação, onde o acionamento da máquina é feito no software ANSYS Twin Builder e seu modelo em elementos finitos é realizado no ANSYS Maxwell. A partir do equacionamento da máquina rotativa são feitas as respectivas equivalências com a máquina linear, que conceberão um modelo no espaço de estados para o atuador considerando um referencial síncrono. No passo seguinte, este modelo será linearizado no entorno de um dado ponto de operação. $\mathrm{O}$ desempenho dos modelos não linear e linearizado será comparado ao obtido pela cossimulação para duas situações distintas de operação: degrau de tensão de quadratura e degrau de distúrbio de força de carga.

\section{MÁQUINA LINEAR SÍNCRONA DE ÍMÃS PERMANENTES}

Esta seção apresenta a descrição da máquina síncrona de ímãs permanentes (MSIP) linear e as equações dinâmicas deduzidas a partir de um modelo de máquina rotativa e convertidas, por analogia, para um modelo de máquina linear.

\subsection{Descrição do atuador}

Este artigo aborda um estudo de caso sobre um atuador eletromagnético linear síncrono de ímãs permanentes, criado para fins de aplicação em suspensão eletromagnética ativa e semiativa, proposto em (Eckert et al., 2018). A topologia da máquina pode ser classificada como atuador eletromagnético linear tubular de bobina longa móvel com duplo arranjo de quase-Halbach, cuja estrutura é apresentada na Figura 1.

Nos arranjos de quase-Halbach, as setas indicam o sentido de magnetização dos ímãs. A movimentação do atuador ocorre no sentido axial $(z)$. O comprimento das culatras $\left(l_{l}\right)$ é de $133,2 \mathrm{~mm}$ e o comprimento da armadura $\left(l_{2}\right)$ é de $212,3 \mathrm{~mm}$. O diâmetro externo da máquina (culatra externa) é de $84 \mathrm{~mm}$ e o diâmetro da bobina é de $64,4 \mathrm{~mm}$. Informações mais detalhadas sobre dimensões e características magnéticas dos materiais podem ser verificadas em (Eckert et al., 2016).



Fig. 1. Vista frontal, em corte, do atuador eletromagnético linear tubular de bobina móvel com duplo arranjo de quaseHalbach.

\subsection{Modelo dinâmico da MSIP}

A classificação de uma máquina em rotativa ou linear, está associada ao tipo de movimentação da máquina. Qualquer topologia de máquina elétrica rotativa pode ser remodelada geometricamente para ser construída de forma planar ou tubular (Boldea, 2013).

Um método tradicional de modelar dinamicamente uma máquina síncrona é transformar o sistema de coordenadas rotativas $(\mathrm{ABC})$ em coordenadas síncronas $(d q 0)$. As equações que fazem essa transformação de coordenadas $A B C / d q 0$ são conhecidas como transformadas de Clarke e Park. Em ABC, o eixo do sistema de referências das grandezas elétricas é fixo, e as grandezas são variáveis ao longo do tempo, enquanto que em $d q 0$ o eixo de referência gira em função do tempo (modificando o ângulo elétrico) e permitindo que as grandezas elétricas sejam mantidas constantes. A velocidade desse ângulo está relacionada com a posição elétrica da parte móvel da máquina, e difere para as topologias rotativa e linear. Enquanto na máquina rotativa o ângulo elétrico pode ser calculado por $2 \cdot \pi \cdot f \cdot t$, sendo $f$ a frequência de excitação e $t$ o tempo, a posição elétrica em uma máquina linear é função da posição axial da parte móvel em relação ao estator $\left(z_{e}\right)$ e do passo polar $\left(\tau_{p}\right)$, dado por

$$
\chi_{e}=\frac{\pi z_{e}}{\tau_{p}} .
$$

Para se obter a referência em $d q 0$ a partir da referência em $\mathrm{ABC}$

$$
S_{d q 0}=\left[\begin{array}{ccc}
S_{d} & S_{d q} & S_{d 0} \\
S_{q d} & S_{q} & S_{q 0} \\
S_{0 d} & S_{0 q} & S_{0}
\end{array}\right], S_{a b c}=\left[\begin{array}{ccc}
S_{a} & S_{a b} & S_{a c} \\
S_{b a} & S_{b} & S_{b c} \\
S_{c a} & S_{c b} & S_{c}
\end{array}\right],
$$

utiliza-se a relação

$$
S_{d q 0}=T_{d q}{ }^{-1} S_{a b c} T_{d q}
$$

onde a matriz de transformação $T_{d q}$ é dada por

$$
T_{d q}=\left[\begin{array}{ccc}
\cos \chi_{e} & -\sin \chi_{e} & 1 \\
\cos \left(\chi_{e}-\frac{2 \pi}{3}\right) & -\sin \left(\chi_{e}-\frac{2 \pi}{3}\right) & 1 \\
\cos \left(\chi_{e}+\frac{2 \pi}{3}\right) & -\sin \left(\chi_{e}+\frac{2 \pi}{3}\right) & 1
\end{array}\right] .
$$


Para transformação de coordenadas (3) em máquinas lineares, é preciso utilizar a relação (1) em (4) ao invés da relação $\chi_{e R}$ $=2 \cdot \pi \cdot f \cdot t$ de máquinas rotativas.

Do ponto de vista do circuito eletromagnético, após a conversão para coordenadas síncronas, as topologias rotativa e linear são similares. Os circuitos equivalentes de uma MSIP na referência $d q 0$, com a posição mecânica no eixo direto $($ eixo $d$ ) e no eixo em quadratura (eixo $q$ ), são mostrados na Figura 2, respectivamente. Esses circuitos serão utilizados para extração das equações dinâmicas elétricas da máquina rotativa/linear.

Considerando que não ocorra saturação magnética nas culatras, que as perdas magnéticas são desprezíveis, que o fluxo magnético é constante e não sofre mudanças com a temperatura nem em relação ao tempo, é possível apresentar as equações, desenvolvidas para modelagem de máquinas rotativas (Krishnan, 2001), como

$$
\begin{aligned}
& v_{d}(t)=R i_{d}(t)+\frac{d \phi_{d}(t)}{d t}-\omega_{e}(t) \phi_{q}(t) \\
& v_{q}(t)=R i_{q}(t)+\frac{d \phi_{q}(t)}{d t}+\omega_{e}(t) \phi_{d}(t)
\end{aligned}
$$

onde, $v_{d}, v_{q}, i_{d}, i_{q}, \phi_{d}$ e $\phi_{q}$ são respectivamente as tensões, correntes e fluxos magnéticos de eixo direto e em quadratura, e $\omega_{e}$ é a velocidade angular elétrica.

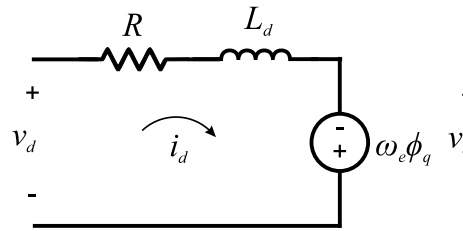

(a)



(b)
Fig. 2. Circuito equivalente da MSIP em (a) posição de eixo direto e (b) posição de eixo em quadratura.

Para uma máquina linear, a onda de tensão induzida se desloca na direção axial, ocasionando uma velocidade elétrica $v_{e}$ diferente da máquina rotativa $\omega_{e}$. Apesar da topologia linear, essa velocidade também é expressa em radianos por segundo, sendo dada por

$$
v_{e}=K_{m} \cdot v_{m}=\frac{p \cdot \pi}{\tau_{p}} \cdot v_{m}
$$

onde $p$ é o número de par de polos e $v_{m}$ a velocidade linear mecânica. Assim, as equações da máquina linear podem ser descritas analogamente as da máquina rotativa em (5) como

$$
\begin{aligned}
& v_{d}(t)=R i_{d}(t)+\frac{d \phi_{d}(t)}{d t}-v_{e}(t) \phi_{q}(t) \mathrm{e} \\
& v_{q}(t)=R i_{q}(t)+\frac{d \phi_{q}(t)}{d t}+v_{e}(t) \phi_{d}(t) .
\end{aligned}
$$

A partir do diagrama de fluxos magnéticos do eixo $d$ e eixo $q$ (Krishnan, 2001), é obtida a relação

$$
\begin{gathered}
\phi_{q}(t)=L_{q} i_{q}(t) \mathrm{e} \\
\phi_{d}(t)=L_{d} i_{d}(t)+\phi_{m g},
\end{gathered}
$$

onde $L_{d}, L_{q}$ e $\phi_{m g}$ representam a indutância de eixo direto, a indutância de eixo em quadratura e o fluxo magnético polar oriundo dos arranjos de ímã permanente, respectivamente. Substituindo (9) e (10) em (7) e manipulando algebricamente chega-se a

$$
\frac{\mathrm{d}}{\mathrm{d} t} i_{d}(t)=\frac{v_{d}(t)-R i_{d}(t)+v_{e}(t) L_{q} i_{q}(t)}{L_{d}} .
$$

De maneira similar, substituindo (9) e (10) em (8), chega-se em

$$
\frac{\mathrm{d}}{\mathrm{d} t} i_{q}(t)=\frac{v_{q}(t)-R i_{q}(t)-v_{e}(t) L_{d} i_{d}(t)-v_{e}(t) \phi_{m g}}{L_{q}} .
$$

As equações (11) e (12) representam a dinâmica das correntes e tensões, na referência síncrona, de uma máquina linear. Da teoria de máquinas elétricas, segue que a velocidade mecânica $\left(v_{m}\right)$ em uma máquina síncrona está diretamente relacionada à sua velocidade elétrica $\left(v_{e}\right)$. Enquanto na máquina rotativa as conversões de velocidade e ângulo mecânicos para velocidade e ângulo elétricos são feitas apenas dividindo pelo número de par de polos $(p)$, nas máquinas lineares essa transformação é dada por

$$
v_{m}=K_{e} \cdot v_{e}=\frac{\tau_{p}}{\pi} \frac{v_{e}}{p} .
$$

A equação típica de conjugado em uma máquina rotativa é

$$
T=J \frac{d \omega_{m}}{d t}+B_{v} \omega_{m}+T_{c},
$$

Onde $J, B_{v}, \omega_{m}$ e $T_{c}$ representam o momento de inércia, o coeficiente de atrito viscoso, a velocidade angular mecânica e o conjugado de carga, respectivamente. Analogamente, a equação mecânica para uma MSIP linear, operando na horizontal, conforme Figura 1, é dada por

$$
F=m \frac{d v_{m}}{d t}+B_{v} v_{m}+F_{c},
$$

onde $m, v_{m}$ e $F_{c}$ representam a massa, a velocidade linear mecânica e a força de carga, respectivamente. É possível verificar em (14) e (15) que o conjugado em uma máquina rotativa é análogo à força no caso linear. Entretanto, para que a força seja dada em newton, as unidades das grandezas são diferentes na máquina linear, o que será mostrado na Seção 5. Além disso, tem-se que a força eletromagnética em uma MSIP linear (Boldea, 2013) é

$$
F=\frac{3}{2} \frac{\pi}{\tau_{p}} p\left(\phi_{m g} i_{q}(t)-\left(L_{d}-L_{q}\right) i_{d}(t) i_{q}(t)\right) .
$$

Supondo que a máquina considerada possua indutâncias síncronas aproximadamente iguais, essa equação pode ser reduzida para

$$
F=\frac{3}{2} \frac{\pi}{\tau_{p}} p \phi_{m g} i_{q}(t) .
$$

Substituindo-se (17) em (15) chega-se a

$$
\frac{\mathrm{d}}{\mathrm{d} t} v_{e}(t)=\frac{\pi p}{\tau_{p} m}\left(\frac{3}{2} \frac{\pi}{\tau_{p}} p \phi_{m g} i_{q}(t)-\frac{B_{v} v_{e}(t) \tau_{p}}{\pi p}-F_{C}\right) .
$$

A equação (18) representa a dinâmica da velocidade e movimentação do motor linear na coordenada de referência síncrona. 


\section{MODELO NO ESPAÇO DE ESTADOS}

O modelo em espaço de estados permite uma descrição interna do sistema. A partir da seção anterior segue que, as equações dinâmicas do MSIP linear na referência $d q 0$ são (11), (12) e (18). Para analisar o modelo em espaço de estados, será considerado um sistema com três estados, sendo eles

$$
x=\left[\begin{array}{lll}
x_{1} & x_{2} & x_{3}
\end{array}\right]^{T}=\left[\begin{array}{lll}
i_{d} & i_{q} & v_{e}
\end{array}\right]^{T},
$$

duas entradas de tensão,

$$
u=\left[\begin{array}{ll}
u_{1} & u_{2}
\end{array}\right]^{T}=\left[\begin{array}{ll}
v_{d} & v_{q}
\end{array}\right]^{T},
$$

e terá como saída os próprios estados, ou seja,

$$
y=\left[\begin{array}{lll}
i_{d} & i_{q} & v_{e}
\end{array}\right]^{T} .
$$

Além disso, o sistema está sujeito a um sinal exógeno de distúrbio causado por $F_{c}(t)$. É possível visualizar que existe produto entre estados em (11) e (12), e isso faz com que o sistema se torne não linear. Com isso (11), (12) e (18) podem ser escritos na forma

$$
\begin{aligned}
& \dot{x}(t)=f\left(x, u, F_{c}\right), \\
& y(t)=g\left(x, u, F_{c}\right)
\end{aligned}
$$

onde

$$
\begin{aligned}
& f\left(x, u, F_{c}\right)=\left[\begin{array}{c}
\frac{1}{L_{d}}\left(u_{1}-R x_{1}+L_{q} x_{2} x_{3}\right) \\
\frac{1}{L_{q}}\left(u_{2}-R x_{2}-L_{d} x_{1} x_{3}-\phi_{m g} x_{3}\right) \\
\frac{\pi p}{\tau_{p} m}\left(\frac{3}{2} \frac{\pi}{\tau_{p}} p \phi_{m g} x_{2}-\frac{B_{v} \tau_{p} x_{3}}{\pi p}-F_{C}\right)
\end{array}\right] \mathrm{e} \\
& g\left(x, u, F_{c}\right)=x .
\end{aligned}
$$

Uma estratégia usual é obter uma aproximação linear da dinâmica deste sistema no entorno de um ponto de operação $(X e, U e)$. Assumindo um dado conjunto de estados de equilíbrio $X e=\left[\begin{array}{lll}i_{d 0} & i_{q 0} & v_{e 0}\end{array}\right]^{\prime}$, os valores de $U e=\left[\begin{array}{ll}v_{d 0} & v_{q 0}\end{array}\right]^{\prime}$ e $F c e$ devem ser tais que $f(X e, U e, F c e)=0$. Assim, o sistema (23) modelado para pequenas variações no entorno do ponto $(X e, U e, F c e)$, isto é, $x(t)=X e+\delta x(t), u(t)=U e+\delta u(t)$ e $F_{c}(t)=F c e+\delta F_{c}(t)$, é representado na forma

$$
\begin{aligned}
& \dot{\delta} \dot{x}(t)=A_{e} \delta x(t)+B_{e} \delta u(t)+B_{d e} \delta F_{c}(t) \\
& \delta y(t)=C_{e} \delta x(t)+D_{e} \delta u(t)+D_{d e} \delta F_{c}(t)
\end{aligned},
$$

onde

$$
\begin{aligned}
& A_{e}=\left.\frac{\partial f}{\partial x}\right|_{x=X e, u=U e, F c=F c e}, B_{e}=\left.\frac{\partial f}{\partial u}\right|_{x=X e, u=U e, F c=F c e} \\
& B_{d e}=\left.\frac{\partial f}{\partial F_{c}}\right|_{x=X e, u=U e, F c=F c e}, C_{e}=\left.\frac{\partial g}{\partial x}\right|_{x=X e, u=U e, F c=F c e} . \\
& D_{e}=\left.\frac{\partial g}{\partial u}\right|_{x=X e, u=U e, F c=F c e}, D_{d e}=\left.\frac{\partial g}{\partial F_{c}}\right|_{x=X e, u=U e, F c=F c e}
\end{aligned}
$$

Com este procedimento nas funções $f\left(x, u, F_{c}\right)$ e $g\left(x, u, F_{c}\right)$ em (22), segue que
$A_{e}=\left[\begin{array}{ccc}-R / L_{d} & v_{e 0} \cdot L_{q} / L_{d} & i_{q 0} \cdot L_{q} / L_{d} \\ -v_{e 0} \cdot L_{d} / L_{q} & -R / L_{q} & -L_{d} \cdot i_{d 0}-\phi_{m g} / L_{q} \\ 0 & 3 \pi^{2} p^{2} \phi_{m g} / 2 \tau_{p}{ }^{2} m & -B_{v} / m\end{array}\right]$,

$$
\begin{gathered}
\text { e } B_{e}=\left[\begin{array}{cc}
1 / L_{d} & 0 \\
0 & 1 / L_{q} \\
0 & 0
\end{array}\right], \quad B_{d e}=\left[\begin{array}{c}
0 \\
0 \\
-\frac{\pi p}{\tau_{p} m}
\end{array}\right], \\
C_{e}=\left[\begin{array}{lll}
1 & 0 & 0 \\
0 & 1 & 0 \\
0 & 0 & 1
\end{array}\right], \quad D_{e}=D_{d e}=[0] .
\end{gathered}
$$

Tendo em vista as simulações que serão apresentadas na Seção 5, seque que os pontos de operação escolhidos são dados por $i_{d 0}=0 \mathrm{~A}, i_{q 0}=v_{q} / R=0,785 \mathrm{~A}$, e uma velocidade elétrica de excitação da máquina $v_{e 0}$ de $50 \mathrm{rad} / \mathrm{s}$. As respostas em malha aberta dos modelos não linear (22) e linearizado (24) serão mostradas na seção de discussão dos resultados, onde será feita uma comparação com os resultados do modelo em elementos finitos.

\section{MODELO DE COSSIMULAÇÃO}

Neste trabalho, o software utilizado para aplicação do MEF é o ANSYS Maxwell Electromagnetics ${ }^{\circledR}$, que resolve problemas eletromagnéticos aplicando as equações de Maxwell na forma diferencial para uma região finita com condições de contorno apropriadas. Como a topologia do atuador é tubular, é possível fazer uma simulação bidimensional, selecionando a opção de simetria em torno de um eixo. Os materiais utilizados no atuador podem ser consultados em (Eckert et al., 2018). Foram impostas na região de contorno do modelo as condições de Dirichlet (potencial vetor magnético igual à zero). O tamanho da região que abrange o modelo é alterado dinamicamente conforme a posição da bobina é alterada. Já as posições dos ímãs e das culatras são mantidas estáticas. $\mathrm{O}$ comprimento dessa região na direção axial se mantém em $100 \%$ do comprimento axial total. Já na direção radial, o comprimento da região é de $300 \%$ do raio externo do atuador. O tamanho máximo de elemento da malha dos $(3 \mathrm{~mm})$ foi escolhido empiricamente de forma a obter um tempo razoável de simulação mantendo a precisão desejada nos resultados.

A próxima etapa do método é a definição da excitação do atuador. O acionamento foi realizado em um simulador de circuitos (ANSYS Twin Builder) da mesma fabricante do software que realiza o cálculo por MEF. Dessa maneira é possível realizar uma cossimulação entre o analisador de circuitos (que realiza a excitação) e o modelo em elementos finitos (que produz a resposta de força, corrente, posição, etc. correspondente à excitação). Dentro do Twin Builder também são adicionadas as características mecânicas do atuador, como massa e atrito dos rolamentos. A Figura 3 mostra o diagrama de cossimulação entre atuador (Maxwell) e sua respectiva alimentação (Twin Builder). 
É possível verificar na Figura 3 que o acionamento é feito por tensão aplicada diretamente nos terminais da máquina. No eixo da máquina (movimento) é definida a massa da parte móvel, atrito dos rolamentos, e são adicionadas uma medição de posição e de velocidade, e também é adicionada uma fonte de força que é o distúrbio de carga. A medida da posição mecânica do eixo é transformada para posição elétrica da transformada de coordenadas, como em (1). Esse valor serve para definir a posição elétrica correta para a transformada de coordenadas rotativa/síncrona e vice-versa.



Fig. 3. Diagrama mostrando como a excitação é feita no atuador (MEF) por meio do simulador de circuitos.

O modelo em elementos finitos foi amplamente explorado e validado em relação ao protótipo real do atuador em (Eckert et al., 2018) e em (Boff et al., 2017), onde os dados de fluxo magnético e indutâncias numéricos foram confrontados com dados experimentais. Sendo assim, o modelo é válido para servir de parâmetro para as comparações subsequentes.

\section{ANÁLISE DOS RESULTADOS}

Nesta seção são apresentados e discutidos os resultados obtidos a partir dos modelos resultantes das Seções 3 e 4 . As análises foram divididas em dois estudos de caso. No caso 1 foram impostas entradas de tensão em quadratura e as saídas do atuador foram analisadas, tanto para o modelo utilizando o método dos elementos finitos (MEF) quanto para o caso descrito no espaço de estados com o modelo linearizado (ML) e não linear (MNL). No caso 2 foram impostas as mesmas condições de tensão em quadratura adicionadas a um distúrbio de força $\left(F_{c}\right)$, sendo analisadas as mesmas saídas do caso 1. Os parâmetros do atuador que serão utilizados na modelagem dinâmica são os mesmos mostrados na Tabela 1.

Os valores de indutância apresentados representam uma média do valor dessas indutâncias (pois elas são variáveis em função da posição axial devido ao efeito de extremidade). No modelo em elementos finitos, os únicos valores atribuídos no Twin Builder são a massa da parte móvel, o atrito dos rolamentos e a resistência por fase. Demais valores são calculados automaticamente pelo software.

\subsection{Caso 1}

As entradas de tensão foram configuradas para um degrau de $v_{d}=0$ e $v_{q}=10 \mathrm{~V}$ no tempo de $5 \mathrm{~ms}$. Como a tensão de eixo direto não produz força (17) e deslocamento, sua entrada foi considerada nula. Enquanto nos modelos linearizado e não linear as entradas são diretamente em $d q 0$, no MEF as referências de tensão são transformadas para o sistema $\mathrm{ABC}$ para serem injetadas no atuador trifásico (Figura 3). Essa conversão de coordenadas é feita com o valor da posição do atuador, como em (1). Isso significa que esse valor é atualizado conforme a posição axial se modifica, para manter sempre o nível de tensão em quadratura fixo. As correntes do atuador são medidas em $\mathrm{ABC}$ e são convertidas ao $d q 0$ para fins de comparação com o ML e MNL. O tempo total de simulação foi de $20 \mathrm{~ms}$. Os resultados desse caso são mostrados na Figura 4.

Tabela 1. Parâmetros do atuador

\begin{tabular}{llll}
\hline \hline \multicolumn{1}{c}{ Descrição } & Símbolo & \multicolumn{1}{c}{ Valor } \\
\hline Passo polar & $\tau_{p}$ & $26,64 \mathrm{~mm}$ \\
Indutância de eixo direto & $L_{d}$ & $8,2884 \mathrm{mH}$ \\
Indutância de eixo em & $L_{q}$ & $8,3972 \mathrm{mH}$ \\
quadratura & $R$ & $12,74 \Omega$ \\
Resistência por fase & $\phi_{m g}$ & $0,1994 \mathrm{~Wb}$ \\
Fluxo magnético polar & $m$ & $1,5 \mathrm{~kg}$ \\
Massa da parte móvel & $m$ & $0,024 \mathrm{Ns} / \mathrm{m}$ \\
Atrito dos rolamentos & $B_{v}$ & 3 \\
Par de polos & $p$ & \\
\hline \hline
\end{tabular}

\subsection{Caso 2}

As entradas de tensão foram mantidas para um degrau de $v_{d}=$ 0 e $v_{q}=10 \mathrm{~V}$ no tempo de $5 \mathrm{~ms}$, porém, um degrau de força de carga $\left(F_{c}\right)$ de $50 \mathrm{~N}$ foi aplicado no instante de tempo de 50 ms. O tempo total de simulação foi aumentado para $100 \mathrm{~ms}$. Os resultados desse caso são mostrados na Figura 5. Essa simulação permite visualizar como o atuador se comporta frente a um distúrbio de carga.

\subsection{Discussão}

Através dos gráficos das Figuras 4 e 5 é possível perceber, de um modo geral, que o modelo em espaço de estados é capaz de replicar as características fundamentais do atuador simulado em elementos finitos, principalmente a dinâmica do ponto de vista da resposta transitória. Nos gráficos de corrente de eixo direto, enquanto no $\mathrm{MEF}$ o valor em regime é contínuo próximo de zero $\left(4,5 \cdot 10^{-3} \mathrm{~A}\right)$, no MNL esse valor é aproximadamente zero $\left(1,8.10^{-4} \mathrm{~A}\right)$ e no ML possui um valor um pouco mais elevado $\left(2,6.10^{-2} \mathrm{~A}\right)$ porém desprezível se comparado aos valores da corrente de eixo em quadratura. Os valores de corrente de eixo direto próximos de zero indicam que o atuador está sendo corretamente acionado na posição de quadratura. A corrente de quadratura é similar nos três modelos, inicialmente um valor mais elevado é exigido para retirar o atuador da inércia e após isso esse valor diminui. $\mathrm{O}$ valor máximo da corrente de quadratura no MEF é 0,582 A, no ML é 0,574 A e no MNL é 0,580 A. O valor dessa corrente no MEF não se aproxima tanto de zero em 
regime $\left(3,4.10^{-2} \mathrm{~A}\right)$ quanto no $\mathrm{ML}\left(2,7.10^{-3} \mathrm{~A}\right)$ e $\mathrm{MNL}$ $\left(3,3 \cdot 10^{-3} \mathrm{~A}\right)$. Isso pode ser explicado pelo fato de que no MEF, o modelo real da máquina é representado considerando parâmetros não lineares, como variação de indutâncias, efeitos de extremidade, fluxo magnético disperso e efeitos de tensões induzidas na armadura causadas pela velocidade. Como a força desenvolvida é em função da corrente $i_{q}(17)$, a ondulação também pode ser identificada no gráfico da força. O mesmo efeito é verificado sutilmente na velocidade elétrica, ou seja, o ML e o MNL tendem a um valor constante em regime permanente (respectivamente, 50,15 e 50,19 rad/s) enquanto o modelo em elementos finitos apresenta uma pequena oscilação no entorno deste valor (média de 50,25 $\mathrm{rad} / \mathrm{s}$ no intervalo de 15 a $20 \mathrm{~ms}$ ).

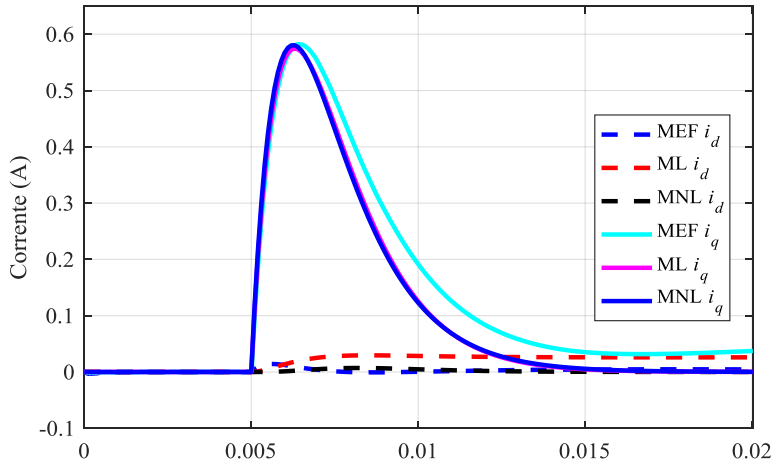

(a) Tempo (s)

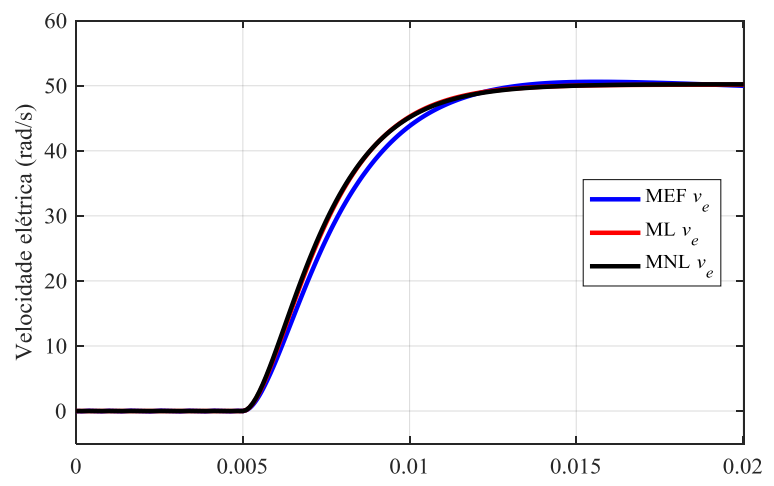

(b) Tempo (s)

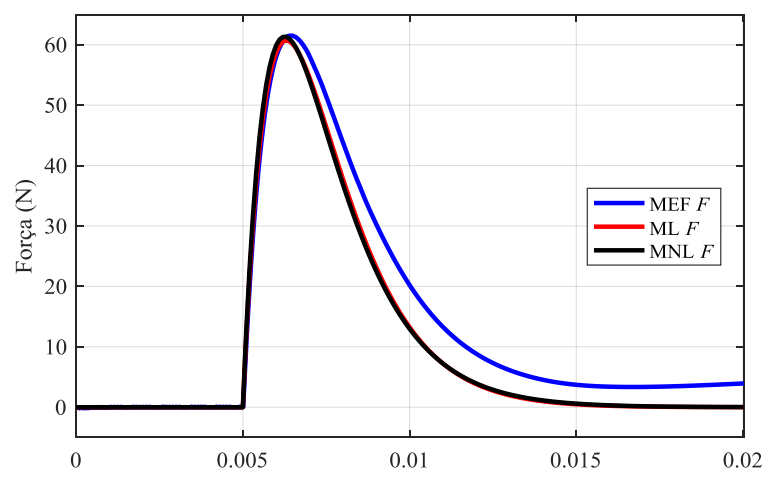

(c) Tempo (s)

Fig. 4. Resultados numéricos do modelo utilizando método dos elementos finitos (MEF) e analíticos do espaço de estados linearizado (ML) e não linear (MNL) para (a) corrente de eixo direto e corrente de quadratura, (b) velocidade elétrica e (c) força eletromagnética, em um tempo de simulação de $0,02 \mathrm{~s}$.
De modo a verificar quantitativamente as similaridades entre a análise por MEF, ML e MNL, a Tabela 2 mostra o coeficiente de correlação dos dados referentes ao caso 1. A análise de correlação identifica a relação entre os dados de duas variáveis, ou seja, ela avalia quanto os dados, de dois modelos selecionados para comparação, estão relacionados (Devore, 2011). Essa correlação pode ser expressa através do coeficiente de correlação de Pearson $(\rho)$. Quando $\rho$ se aproxima de $100 \%$ a relação entre as duas variáveis é forte, e quanto mais próximo de zero a relação se torna mais fraca.

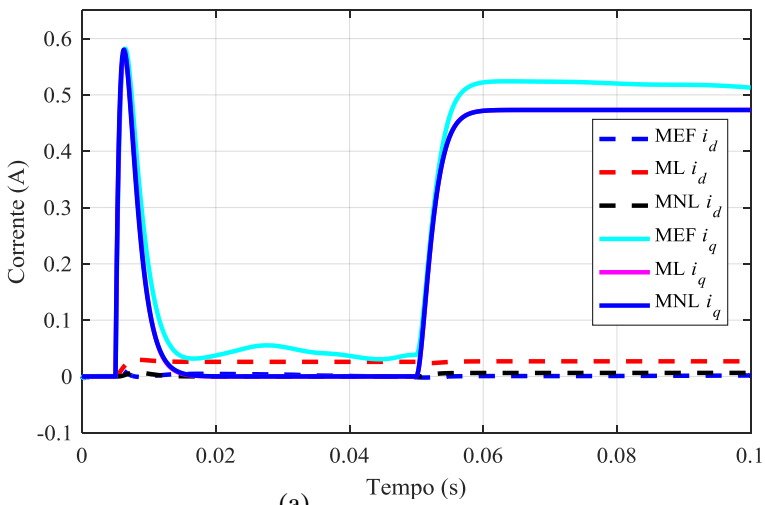

(a)

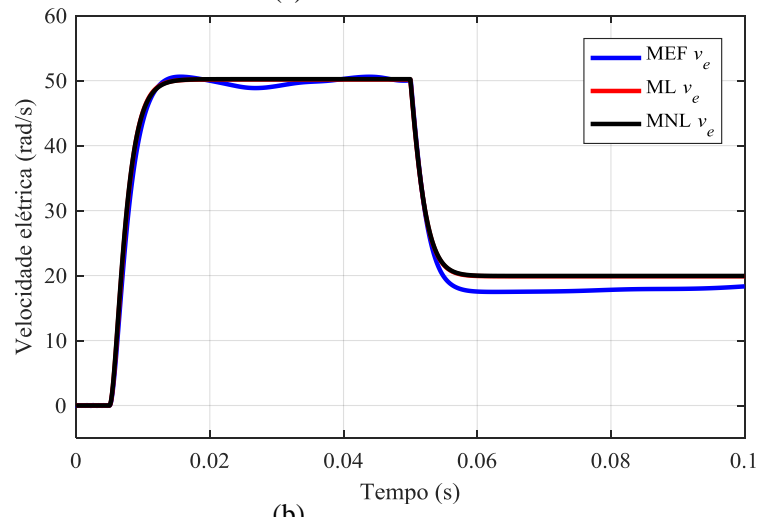

(b)



Fig. 5. Resultados numéricos do modelo utilizando MEF e analíticos do ML e MNL para (a) corrente de eixo direto e quadratura, (b) velocidade elétrica e (c) força, em um tempo de simulação de $0,1 \mathrm{~s}$ onde um distúrbio de força de $50 \mathrm{~N}$ é aplicado em $0,05 \mathrm{~s}$.

Analisando a Tabela 2 é possível verificar que a correlação é fraca para o parâmetro corrente de eixo direto $\left(i_{d}\right)$, e é extremamente forte para os demais parâmetros avaliados $\left(i_{q}\right.$, $v_{e}$ e $F$ ). O baixo nível de correlação de $i_{d}$ pode ser explicado devido aos transitórios dos modelos serem relativamente 
distintos. Considerando a operação em regime permanente, no intervalo de 15 a $20 \mathrm{~ms}$, os coeficientes de correlação de $i_{d}$ ficam entre 95 e $99 \%$ para todos os modelos. A diferença entre o ML e MNL se mostrou muito pequena, portanto é possível concluir que o ML representa bem o sistema nessa faixa de operação.

\section{Tabela 2. Estatística dos dados - Caso 1}

\begin{tabular}{|c|c|c|c|c|c|}
\hline \multirow[b]{2}{*}{ Análise } & \multirow[b]{2}{*}{ Estatística } & \multicolumn{4}{|c|}{ Grandezas analisadas } \\
\hline & & $i_{d}$ & $i_{q}$ & $v_{e}$ & $F$ \\
\hline $\mathrm{MEF} / \mathrm{ML}$ & Coeficiente de & 26,31 & 99,11 & 99,90 & 99,11 \\
\hline MEF/MNL & Correlação de & 18,14 & 98,87 & 99,89 & 98,87 \\
\hline ML/MNL & Pearson (\%) & 54,97 & 99,97 & 99,99 & 99,97 \\
\hline
\end{tabular}

Para fins de verificação da adequação do ML e do MNL frente a um distúrbio de força, em relação ao MEF, o caso 2 foi desenvolvido. Através da Figura 5, é possível perceber que quando uma força é aplicada no eixo do atuador de maneira linear, este se comporta como um gerador. Correntes são geradas, majoritariamente na posição de quadratura, e existe uma redução da velocidade, que dependendo da força de carga pode se tornar negativa. Isso causaria uma velocidade constante e negativa. $O$ sinal que indica velocidade negativa deve-se ao fato que, em um gerador, a força eletromagnética tem sentido oposto à velocidade.

Para ambos os casos, é visível que quando o sistema está em repouso, é necessária uma corrente maior para tirar o sistema da inércia, a partir daí existe uma corrente mínima apenas para tracionar a máquina perante o atrito dos rolamentos. No caso 2, em $50 \mathrm{~ms}$ são inseridos $50 \mathrm{~N}$ como distúrbio, fazendo com que a máquina gere a corrente apropriada em eixo de quadratura para tal. Como a tensão de acionamento do atuador consegue produzir uma corrente suficiente para compensar esse distúrbio, o atuador consegue compensar essa força e continua seu deslocamento. Entretanto, a velocidade é reduzida. Para uma saída de velocidade/posição regulada no valor desejado, é necessário o desenvolvimento de um controle de velocidade ou posição em malha fechada, onde o controlador faz as compensações necessárias de modo a manter erro nulo entre referência e variável controlada. Considerando os testes realizados nesta seção, o modelo linearizado representa bem o sistema e está adequado a ser utilizado para esses fins.

\section{CONCLUSÕES}

Este trabalho apresentou a validação do modelo de um atuador eletromagnético linear tubular. O modelo foi validado comparando a modelagem dinâmica no espaço de estados linearizado e não linear com uma cossimulação transiente. A cossimulação entre circuito de acionamento e modelo da máquina em elementos finitos se mostrou uma ferramenta importante para o estudo de atuadores lineares, pois, ela pode ser utilizada para a validação de modelos dinâmicos. Essa etapa pode substituir a validação experimental ou complementar o modelo dinâmico analítico antes dos testes experimentais. A comparação entre os modelos não linear e linearizado mostrou diferenças pouco significativas na faixa de operação desejada. Assim, concluise que este modelo é adequado e pode ser utilizado no projeto de controladores em malha fechada visando à regulação de velocidade e ou posição do atuador.

\section{AGRADECIMENTOS}

O presente trabalho foi realizado com apoio da Coordenação de Aperfeiçoamento de Pessoal de Nível Superior - Brasil (CAPES) - Código de Financiamento 001. Este trabalho foi financiado em parte pela FAPERGS (17/2551-0000897-9), e em parte pelo Conselho Nacional de Desenvolvimento Científico e Tecnológico $(\mathrm{CNPq})$ por meio de concessão de bolsa de doutorado.

\section{REFERÊNCIAS}

Boff, B. H. B. et al. (2017) 'Influence of End Effects on Direct- and Quadrature-Axis Inductances in Linear Electromagnetic Actuators', IEEE Transactions on Magnetics, 53(11), pp. 1-7.

Boldea, I. (2013) Linear Electric Machines, Drives, and MAGLEVs Handbook. Boca Raton: CRC Press.

Devore, J. L. (2011) Probability and Statistics for Engineering and the Sciences. 8th edn, Technometrics. 8th edn. Boston: Cengage Learning.

Eckert, P. R. et al. (2016) 'Design methodology of a dualhalbach array linear actuator with thermalelectromagnetic coupling', Sensors (Switzerland), 16(3), p. 360 .

Eckert, P. R. et al. (2018) 'Dual Quasi-Halbach Linear Tubular Actuator With Coreless Moving-Coil for Semiactive and Active Suspension', IEEE Transactions on Industrial Electronics, 65(12), pp. 9873-9883.

Fang, S. et al. (2015) 'A Novel Flux Weakening Control Strategy for Permanent Magnet Actuator of Vacuum Circuit Breaker', IEEE Transactions on Industrial Electronics, pp. 1-1.

Krishnan, R. (2001) Electric Motor Drives: Modeling, Analysis, and Control. 1st edn. Upper Saddle River: Prentice Hall.

Liang, W. et al. (2014) 'A New Method for Multiple FiniteElement Models in Cosimulation With Electrical Circuit Using Machine Multiloop Modeling Scheme', IEEE Transactions on Industrial Electronics, 61(12), pp. 6583-6590.

Zanatta, A. P. et al. (2018) 'Tubular linear permanent magnet synchronous machine applied to semi-active suspension systems', COMPEL - The International Journal for Computation and Mathematics in Electrical and Electronic Engineering, 37(5).

Zhu, X. et al. (2007) 'A Transient Cosimulation Approach to Performance Analysis of Hybrid Excited Doubly Salient Machine Considering Indirect Field-Circuit Coupling', IEEE Transactions on Magnetics, 43(6), pp. 2558-2560. 